\title{
Progress in EUV resist Performance
}

\author{
A.M. Goethals, R. Gronheid, F. Van Roey, H. H. Solak* and Y. Ekinci* \\ IMEC, Kapeldreef 75, 3001 Leuven, Belgium \\ ${ }^{*}$ Laboratory for Micro and Nanotechnology, Paul Scherrer Institute, Villigen CH-5232, Switzerland
}

\begin{abstract}
Extreme ultraviolet lithography (EUVL) is the preferred solution for the $32 \mathrm{~nm}$ node. This paper reports on EUV resist screening results by EUV Interference Lithography, targeting at $32 \mathrm{~nm}$ half pitch resolution. Progress in resist resolution, sensitivity and LER has been made. Champion results for chemically amplified resists are $30 \mathrm{~nm}$ resolution, $3.8 \mathrm{~nm}(3 \sigma)$ LER and $<10 \mathrm{~mJ} / \mathrm{cm}^{2}$ sensitivity. However these were not accomplished in one single resist. The performance was compared to imaging in non-chemically amplified PMMA resist showing higher resolution and improved LER $(2.5 \mathrm{~nm})$ at the expense of photospeed. On full field scanners the resist performance might be dominated by the flare. Possible impact of flare on resist profiles has been studied through exposures on a MicroExposure Tool and through contrast demodulation experiments with the interference set-up. Increasing the surface inhibition in the resist is suggested as a way to cope with the higher flare levels of full field scanners.
\end{abstract}

Keywords: EUV lithography, EUV resists, interference lithography, resist sensitivity, line edge roughness, flare

\section{Introduction}

Extreme ultraviolet lithography is being developed for introduction at the $32 \mathrm{~nm}$ node. Infrastructure development such as exposure tools, reticles, resists, light source is well underway. The world's first 0.25 NA EUV full field scanner has been built and initial imaging performance results were reported by ASML [1]. IMEC will install such a full-field EUV pre-production tool from ASML in its $300 \mathrm{~mm}$ research fab later this year (2006).

Following the EUVL symposium held in San Diego in November 2005, the issue of simultaneously addressing resolution, dose, and LER in resists became the number \#1 critical issue for EUVL implementation. Resist development to meet the specifications for the $32 \mathrm{~nm}$ node requires access to EUV exposure tools with resolution beyond $32 \mathrm{~nm}$. There are very few EUV instruments available in the field for resist screening. The EUV Interference lithography setup [2-5] in the Paul Scherrer Institute (PSI) in Switzerland as well as small field Micro-exposure tools (MET) with an NA of 0.3 as installed in the US [6-9] and in Japan [10-11] are effectively used for evaluating the performance of candidate EUV resist materials.

In this paper, results are presented of EUV resist imaging performance based on interference lithography in preparation of a resist process for our ASML Alpha Demo Tool (ADT). EUV interference lithography, by its superior aerial image contrast, provides excellent resolution to use the resists towards resolution limits. However, flare is intrinsically high in EUV and especially on the first full field scanners, with $>10 \%$ flare values reported [1], this may dominate the resist performance. Therefore, the impact of flare on the resist profiles has been studied through exposures on a MET system and through contrast demodulation experiments on the interference setup.

\section{Experimental conditions}

EUV interference exposures were done at the Paul Scherrer Institute (PSI). The interference setup at PSI uses transmissive gratings (mask) and a synchrotron light source. The working principle of this set-up has previously been described [2] and it has proven to be an excellent instrument for early 
resist testing due to its superior resolution capabilities [3-5]. Experiments were also carried out on the small-field MET at the Advanced Light Source (ALS) synchrotron in Berkeley [7]. This instrument is capable of printing $200 \times 600 \mu \mathrm{m}$ field size and is equipped with $0.3 \mathrm{NA} 5 \mathrm{X}$ reductions optics. Annular $(\sigma=0.35-0.55)$ illumination was used for all exposures.

All experiments were carried out using EUV resists supplied by commercial vendors.

Chemically amplified resists were coated at 80-120nm film thickness on 8" (PSI) or 4" (MET) HMDS-primed silicon wafers. Vendor recommended settings for post-apply bake and post-exposure bake and development were used. At both facilities stand-alone coaters and bake plates were used. At the ALS/MET wafer handling is done in an amine-filtered environment to avoid molecular contamination. At PSI no preventive measures for amine contamination during coating or baking are taken and the level of airborne amine contamination is not known. However, after exposure the vacuum chamber is vented with high purity nitrogen and a hotplate is located next to the exposure tool in order to minimize post exposure delay. In practice all samples are baked within $30 \mathrm{sec}$ after they leave the exposure chamber. Moreover, a $\mathrm{N}_{2}$-curtain at the hotplate has been installed recently in order to limit contamination effects during PEB.

The CD measurements on the 8" wafers were carried out using a KLA XP8100 top-down scanning electron microscope (SEM). Crosssectional micrographs were made using a FEI XL30 or NOVA200 SEM.

For characterization of LER, SEM pictures are taken and analyzed off-line using the software developed by Demokritos [12].

\section{Progress in EUV resists performance using interference lithography}

To follow the progress in EUV resist performance, a number of new chemically amplified resists from different suppliers have been tested on the EUV interference lithography set-up at PSI and the results are compared to previous experiments [3-5].

For several of the resist samples tested the resolution was limited due to T-top formation. This especially was the case for platforms that are more susceptible to amine contamination than PHOST, such as acrylate polymers and molecular resists. Chemical filtration and $\mathrm{N}_{2}$-purging during PEB are proposed as solutions to this problem. The use of $\mathrm{N}_{2}$-purging during PEB resulted in improved performance as is illustrated in Figure 1. Moreover, it appears that the lower contamination also allows patterning of lower resolution with the PHOST chemistry. Installation of chemical filters is also being considered but has not been implemented yet.

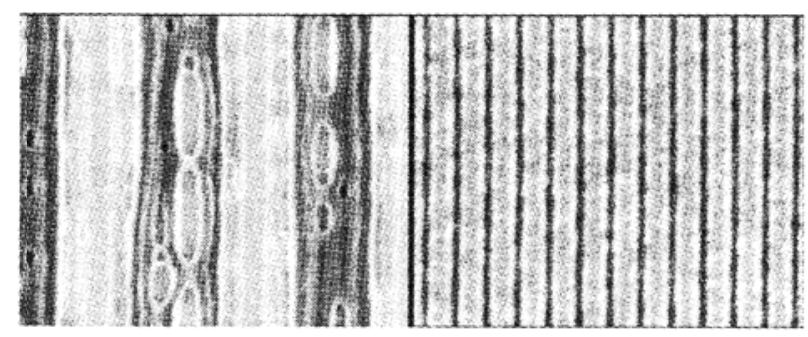

Fig. 1: EUV-24 resist processed without (left) and with (right) $\mathrm{N}_{2}$ purging during $\mathrm{PEB}$

A comparison of the better performing resist samples is illustrated in table I, including the time line when the samples became available. The main performance criteria are sensitivity ( $=$ dose to size for the $50 \mathrm{~nm} \mathrm{L/S}$ ), line edge roughness and ultimate resolution. Resist EUV-18 is considered as the reference (best overall performance last year) to evaluate the progress.

\begin{tabular}{|l|c|c|c|c|}
\hline Resist & dose-50nm US & LER & Resolution & Timing \\
\hline & $\mathbf{m J / c m} \mathbf{2}$ & $\mathbf{3}$ sigma & $\mathbf{n m}$ & \\
\hline EUV-6 & 10.2 & $12.60 \mathrm{~nm}$ & $35-40 \mathrm{~nm}$ & Q4 '04 \\
\hline EUV-18 & 17.5 & $5.43 \mathrm{~nm}$ & $32.5 \mathrm{~nm}$ & Q1 '05 \\
\hline EUV-24 & 17.0 & & $40 \mathrm{~nm}^{*}$ & Q3 '05 \\
\hline EUV-25 & 7.5 & $5.7 \mathrm{~nm}$ & $32.5 \mathrm{~nm}$ & Q3 '05 \\
\hline EUV-26 & 15.3 & & $40 \mathrm{~nm}$ & Q3 '05 \\
\hline EUV-33 & 13.3 & & $40 \mathrm{~nm}^{\star}$ & Q4 '05 \\
\hline EUV-30 & 12.3 & $6.14 \mathrm{~nm}$ & $<32.5$ & Q1 '06 \\
\hline EUV-36 & 21.7 & $3.8 \mathrm{~nm}$ & 35 & Q1 '06 \\
\hline
\end{tabular}

Table I : Overview of resist performance of chemically amplified resists exposed with interference lithography.

The sensitivity of the materials ranges from $7.5 \mathrm{~mJ} / \mathrm{cm}^{2}$ to $>20 \mathrm{~mJ} / \mathrm{cm}^{2}$. The specification for sensitivity being $<10 \mathrm{~mJ} / \mathrm{cm}^{2}$ [13] is only obtained for one of the resists. LER is typically 5 to $6 \mathrm{~nm}$ $(3 \sigma)$ on a nominal $50 \mathrm{~nm} \mathrm{L/S}$ for chemically amplified resist. Resist EUV-36 shows a much lower LER of $3.8 \mathrm{~nm}(3 \sigma)$, however at the expense of a lower sensitivity and lower resolution. Topdown images and $\mathrm{X}$-sectional profiles for the resists are illustrated in Figures 2-5. Modulation down to $25 \mathrm{~nm} \mathrm{~L} / \mathrm{S}$ is seen in several of the stateof-the-art EUV resists (Fig. 2), however when 
looking at the $\mathrm{X}$-sections, real resolution is limited either by incomplete development or top loss. The champion result with regard to resolution is $30 \mathrm{~nm}$ in X-section (Fig. 5) in resist EUV-30 in a resist thickness of $110 \mathrm{~nm}$

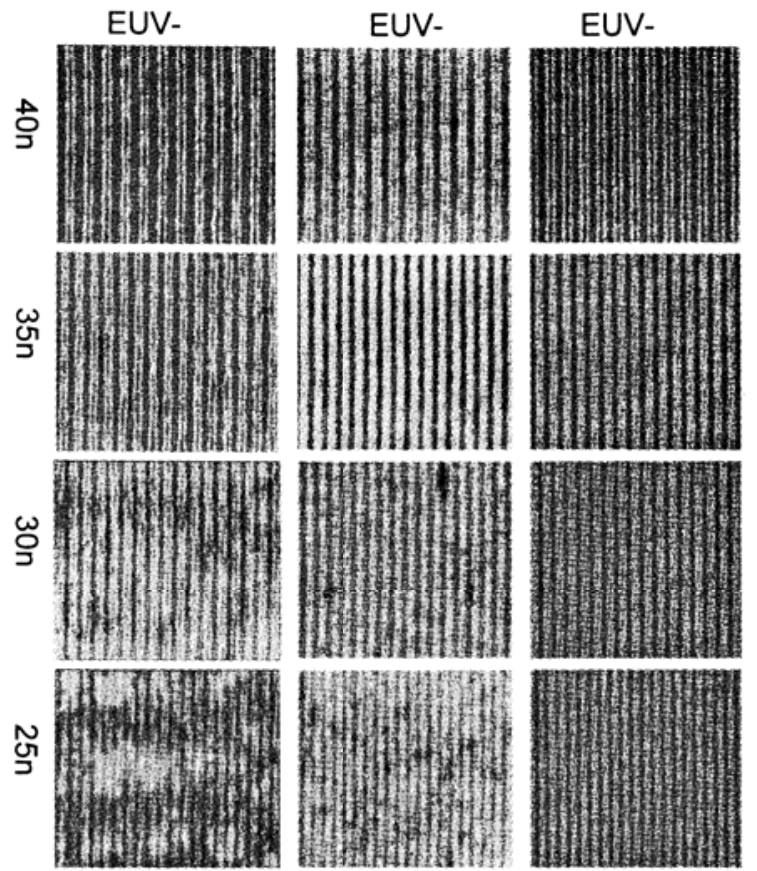

Fig. 2 : Top-down pictures of $40 \mathrm{~nm}$ down to $25 \mathrm{~nm}$ Lines and Spaces in latest state-of-the-art resists

With the improvement in resist performance, pattern collapse (instead of top-erosion) is seen more and more as a resolution limiting factor, (Fig. 3) especially for the (meth)acrylate based backbones. This is the case for the resists EUV-24 and EUV-33, who both show straight profiles down to $40 \mathrm{~nm}$ in a resist thickness $>110 \mathrm{~nm}$. Resist thicknesses will need to be decreased to $<100 \mathrm{~nm}$ for the sub-32nm resolution in future experiments.

In summary, with regard to the reference resist (EUV-18), progress has indeed been made in resolution (resist EUV-30), in sensitivity at the same resolution (EUV-25) and in LER (EUV-36). Moreover, exposure latitude for the newer resist materials such as EUV-30 and EUV-36 is $30 \%$ on nominal $50 \mathrm{~nm} \mathrm{L/S}$. This is a significant improvement with regard to reported values [4] of $12-18 \%$ on the $1^{\text {st }}$ generation materials (EUV-6, EUV-18).

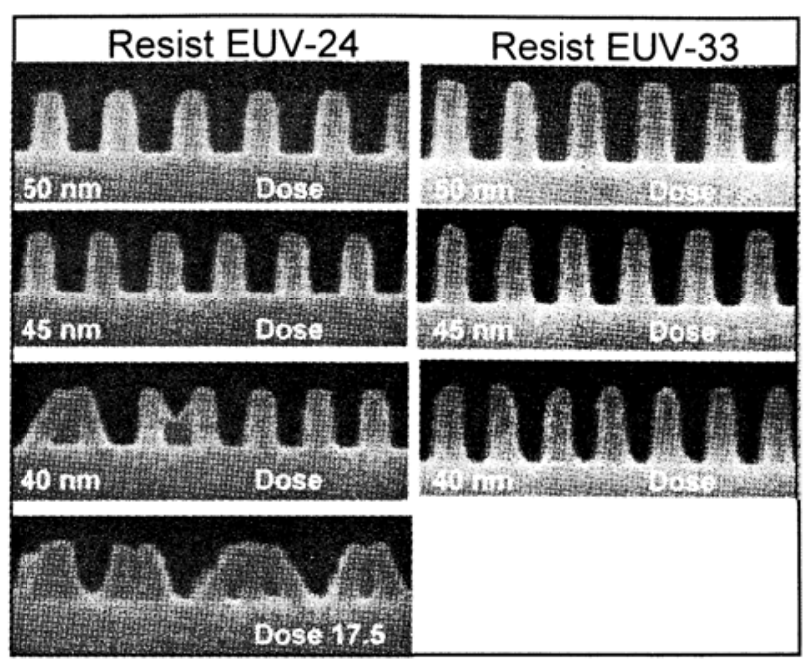

Fig. 3 : X-sectional SEM pictures of resists EUV-24 and EUV-33, 193nm chemistry based and resolution limited by pattern collapse.

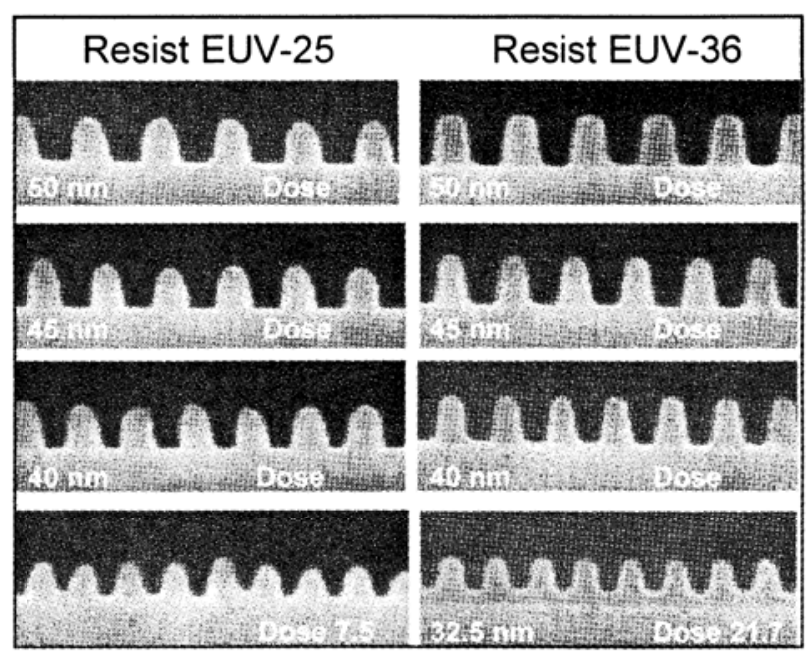

Fig.4 : X-sectional resolution pictures for resists EUV25 and EUV-36.

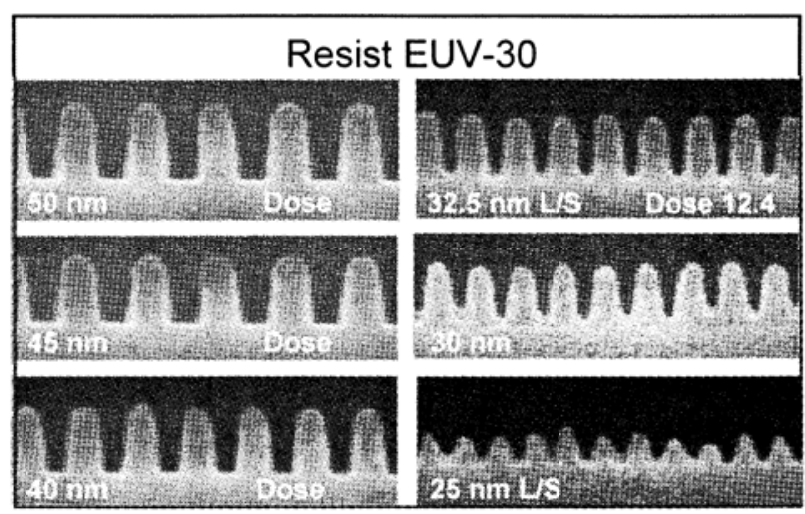

Fig.5 : Champion result with regard to resolution : Xsectional pictures of EUV-30 resist. 
The key issue remaining is still to combine all requirements (sensitivity, low LER, resolution) into one resists.

For sub-32nm node, acid diffusion, blurring the latent image in chemically amplified resist during PEB, will most likely become a resolution limiting factor [14]. Therefore, alternative nonchemically amplified resists are being investigated.

The patterning performance of the non-CAR PMMA has been evaluated using the same mask as was used for the CAR evaluations. Top down and $\mathrm{X}$-section SEM images show the good resolution and pattern profiles in PMMA (Fig. 6). Also LER for PMMA is much better than for all CAR materials that have been screened so far. On $30 \mathrm{~nm}$ $\mathrm{L} / \mathrm{S}$ patterns the LER is $2.5 \mathrm{~nm}(3 \sigma)$. Since this mask is calibrated to the Berkeley MET, the sizing dose of PMMA can be determined. It is surprisingly low $\left(40-50 \mathrm{~mJ} / \mathrm{cm}^{2}\right)$ for a non-CAR under the employed processing conditions. This means that the sizing dose of PMMA is less than an order of magnitude away from the current target. One of the reasons may be that each EUV photon creates several secondary electrons, which give the chain-scissions that cause the solubility change (i.e., EUV gives the amplification 'for free'). Similar to acid diffusion, the secondary electrons give image blurring but the length scale is expected to be roughly an order of magnitude less. This results in improved resolution and a resolution of $17 \mathrm{~nm}$ has indeed been demonstrated $[3,4]$. Further enhancement of the photospeed of PMMA may be possible by changing the process conditions or by some chemical tuning (e.g. to improve the dissolution).
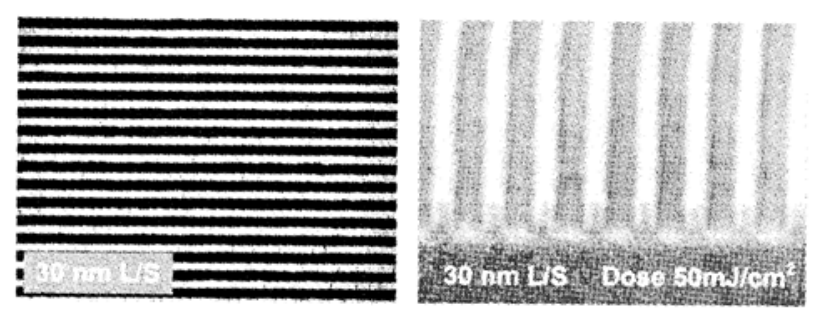

Fig. 6 : Top-down (left) and x-section (right) SEM images for $30 \mathrm{~nm}$ dense line/spaces in PMMA imaged with EUV interference lithography.

\section{Influence of flare on resist performance}

One of the issues an EUV resist will have to cope with is the high EUV flare levels in the full field scanners and the accompanying decreased aerial image contrast. Some information on the performance of EUV resists under higher flare levels had been obtained from exposures at the MET at Berkeley $[5,7,8]$. Comparable results were found in terms of ultimate resolution and exposure latitude but significant differences in profiles were seen between the interference exposures and the MET exposed wafers. The shape and quality of the resist profiles is better with interference lithography (PSI). On the MET, profiles have top loss and show a significant amount of footing (Fig. 7). The difference in profiles (Fig. 7) was mainly attributed to the higher flare levels on the MET.
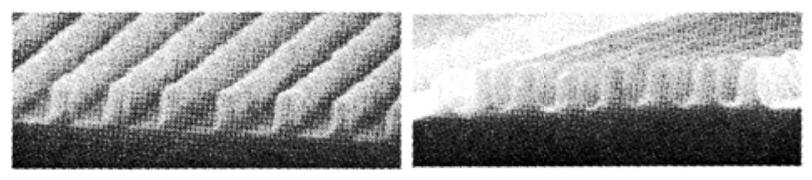

Fig. 7 : X-sectional profiles of 50nm L/S in resist EUV16 , exposed with interference lithography (left) and exposed on the MET at Berkeley (right).

Intentionally increasing the flare on the MET by doing an EUV open frame exposure in addition to the pattern exposure, resulted in a further decrease in contrast and increased footing behaviour (Fig. 8).
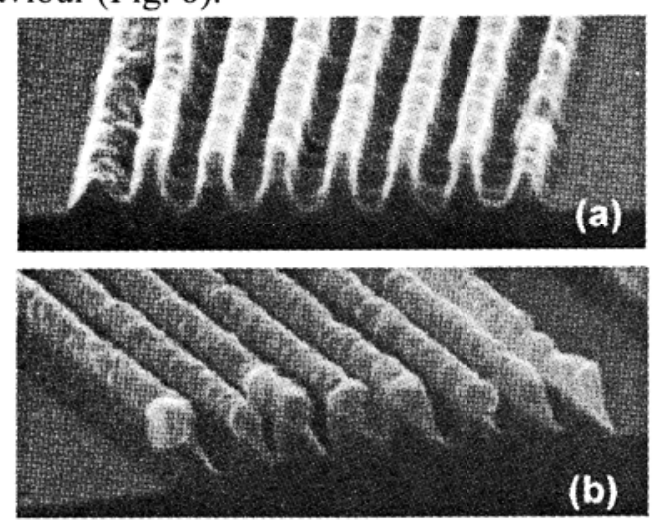

Fig. 8 : X-sectional profiles of 50nm L/S in resist EUV18 , exposed on the MET at Berkeley without additional flare (a) and with 10\% additional flare (b).

To obtain a better correlation with the MET results, contrast demodulation experiments were performed at PSI. EUV open frame exposures were performed over EUV patterned areas. This was done at several dose levels relative to the patterning dose. Effectively, the open frame exposure induces artificially added flare. Interestingly, the effect on the pattern profiles opposed the expected result. Increased T-topping was seen at higher flare levels (Figure 9). 


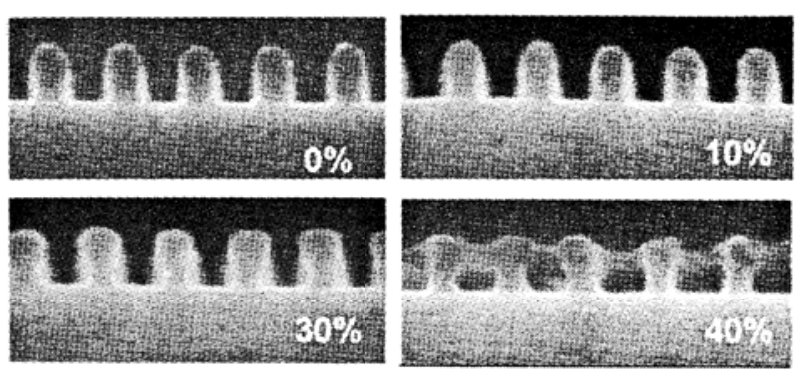

Fig. 9 : Resist profiles in EUV-16 resist obtained from interference exposure with added flare increasing from $0 \%$ to $40 \%$ of the patterning dose.

This result is attributed to chemical contamination at PSI. As a side effect of the added flare, a lower patterning dose is required for the higher flare levels. This makes these patterns more vulnerable for the amine contamination. This explanation is corroborated by KLA Prolith simulations as is shown in Figure 10. In the presence of chemical contamination, T-topping due to surface contamination becomes more pronounced at higher flare levels (Fig. 10, bottom). In the absence of chemical surface contamination, (as is the case for the filtered environment of the MET), simulation predicts more sloped resist sidewalls and resist top loss at increasing flare levels (Fig. 10, top). The latter was experimentally confirmed by the contrast demodulation experiments at the MET.

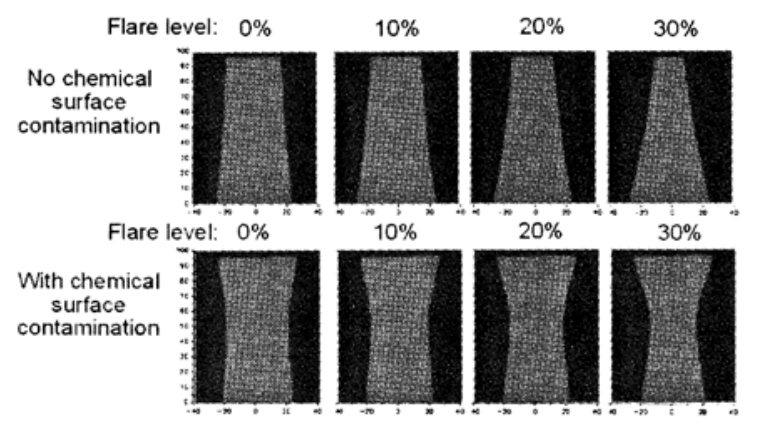

Fig. 10: Prolith simulated pattern profiles at flare levels increasing from 0 to $30 \%$ in the absence (top) and presence (bottom) of chemical contamination.

From this study, it was concluded that the differences in profiles between MET and PSI are caused by the combined effect of different levels of flare and of airborne chemical contamination. Experiments in filtered environment are needed to further confirm this hypothesis with chemically amplified resists at PSI.

The decrease in image contrast by the addition of flare is also expected to have an influence on the Line edge roughness. Line edge roughness was measured for the resist as a function of additional flare as illustrated in Figure 11. Only a minor increase in LER was observed at higher flare levels.

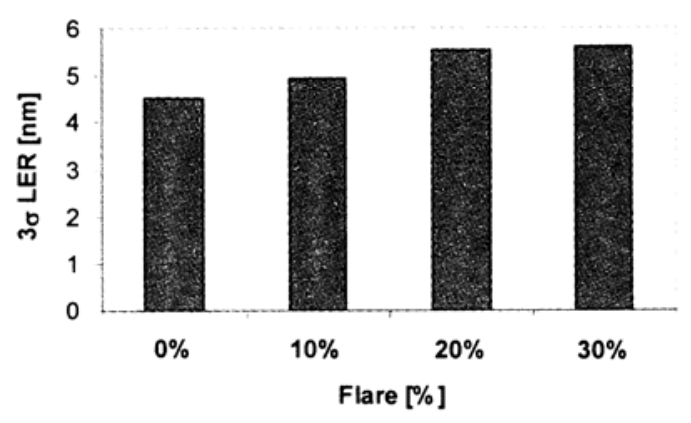

Fig 11 : Line edge roughness for 50nm L/S (resist EUV16) exposed with interference lithography with added flare from $0 \%$ to $30 \%$.

Contrast demodulation experiments with PMMA resist resulted in increased sidewall slope and increased resist top loss at higher flare levels. PMMA resist, as being a non-chemically amplified, is indeed not sensitive to amine contamination and reacts as predicted by the simulation (Fig.10, top).

More surface inhibition will result in resists that are more able to deal with the high flare levels that are expected for the early EUV full field scanners.

\section{Conclusions}

In this work the progress in EUV resist performance, based on interference lithography has been tracked. Patterning of EUV resists at $32 \mathrm{~nm}$ resolution is challenging, but feasible. Today, several chemically amplified resists are capable of resolving $32.5 \mathrm{~nm}$. One material was able to resolve $30 \mathrm{~nm} \mathrm{~L} / \mathrm{S}$ in a $110 \mathrm{~nm}$ thick resist layer at a dose of $12 \mathrm{~mJ} / \mathrm{cm}^{2}$. The champion result for LER is $3.8 \mathrm{~nm}(3 \sigma)$ in a chemically amplified resist. Sensitivities lower than $10 \mathrm{~mJ} / \mathrm{cm}^{2}$ are feasible. However simultaneous achievement of resolution, LER and sensitivity requirements is not accomplished in any of the resists. Further optimization of the chemically amplified resist concept requires careful tuning to find a compromise for the simultaneous optimization of sensitivity, LER and resolution. As an alternative route, non-chemically amplified resists such as PMMA, demonstrating higher resolution and better LER $(2.5 \mathrm{~nm})$, certainly appear to be attractive candidates if their photospeed can be increased sufficiently (about 5 times). 
The performance of resist materials under the high flare conditions that are expected on commercial EUV tools forms an additional concern.

Possible impact of flare on resist profiles has been studied through exposures on a Micro-Exposure Tool and through contrast demodulation experiments with the interference set-up. The shape of the profiles is the result of a combined effect of flare level and of surface inhibition due to airborne chemical contamination. More surface inhibition will result in resists that are more able to deal with the high flare levels that are expected for the early EUV full field scanners.

Such a first full field scanner has been built [1] and ASML reported initial imaging results demonstrating sub-40 $\mathrm{nm}$ resolution in one of the $1^{\text {st }}$ generation resists. The installation of such an ADT tool in IMEC, will enable resist tuning and process optimization for $32 \mathrm{~nm}$ implementation.

\section{Acknowledgements}

The authors are indebted to the European Commission and the $\mathrm{Medea}^{+}$organization, for the funding of the European projects IST- More MOORE and T406 EXCITE respectively. We would like to thank Koen Van Ingen-Schenau (ASML), Cyril Vannuffel and Amandine Jouve (CEA-Leti) for the joint experiments and many discussions within the Excite project. We acknowledge Peter Leunissen at IMEC for the discussions on LER and for providing the analysis of LER measurement. Finally we like to thank the resist suppliers for supplying the materials.

\section{References}

[1] H. Meiling, H. Meijer, V. Banine, R. Moors, R. Groeneveld, H.-J. Voorma, U. Mickan, . Wolschrijn, B. Mertens, G. van Baars, P. Kurz and N. Harned, Proc. SPIE, 6151 (2006), 615108 .
[2] H.H. Solak, C. David, J. Gobrecht, V. Golovkina, F. Cerrina, S.O. Kim, J.F. Nealey, Microelectronic Engineering 67-68 (2003) 5662.

[3] Roel Gronheid, Harun H. Solak, Yasin Ekinci, Amandine Jouve and Frieda Van Roey, Microelectronic Engineering 83 (2006), 11031106

[4] Roel Gronheid, Anne-Marie Goethals ${ }^{1}$, Peter Leunissen', David Van Steenwinckel' ${ }^{2}$, Harun H. Solak, "Resist evaluation using EUV interference lithography", presented at the 3rd International EUVL Symposium, 1-4 November, 2004, Miyazaki, Japan.

[5] A.M. Goethals, R. Gronheid, L.H.A. Leunissen , F. Van Roey, and H.H. Solak, J. Photopolym. Sci. Technol., Vol 18, $\mathrm{N}^{0}$ 5(2005), 647-654.

[6] J. Roberts et all, Microelectronic Engineering 83 (2006) 672-675.

[7] P. P. Naulleau, K.A. Goldberg, E.H. Anderson, J.P. Cain, P. Denham, B. Hoef, K. Jackson, A. Morlens, S. Rekawa, Proc. SPIE, 5751 (2005), 56-63.

[8] P. Naulleau et all, Proc. SPIE, 6151 (2006), $61510 \mathrm{Y}$

[9] K. Lowack, A. Rudack, K. Dean, M. Malloy and M. Lercel,, Proc. SPIE, 6151 (2006), 61512U.

[10] D. Goo, Y. Tanaka, Y. Kikuchi, H. Oizumi, F. Kumasaka,, I. Nishiyama, Proc. SPIE, 6151 (2006), 61512R.

[11] H. Oizumi, F. Kumasaka, Y. Tanaka, T. Hirayama, D. Shiono, H. Hada, J. Onodera, A. Yamaguchi, I. Nishiyama, Microelectronic Engineering 83 (2006) 1107-1110.

[12] V. Constantoudis, G. P. Patsis, L.H.A. Leunissen and E. Gogolides, , Proc. SPIE. 5375, (2004), p. 967-977.

[13] N. Harned, B. Blum, H. Meijer, H. Meiling, P. Kuerz, "Progress on the realization of EUV Lithography", presented at the $4^{\text {th }}$ International EUVL Symposium, 7-10 November, 2005, San Diego, US.

[14] U. Okoroanyanwu, J. Lammers, Future Fab, Vol. 17, (2004), 68-71. 